EOMmun Communication et organisation

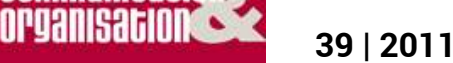

Les applications de la sémiotique à la communication des organisations

\title{
La sémiotique de terrain aujourd'hui, enjeux et propositions
}

Anthony Mathé

\section{OpenEdition}

\section{Journals}

Édition électronique

URL : http://journals.openedition.org/communicationorganisation/3081

DOI : 10.4000/communicationorganisation.3081

ISSN : 1775-3546

Éditeur

Presses universitaires de Bordeaux

Édition imprimée

Date de publication : 1 juin 2011

Pagination : $95-110$

ISBN : 978-2-86781-744-1

ISSN : $1168-5549$

Référence électronique

Anthony Mathé, "La sémiotique de terrain aujourd'hui, enjeux et propositions », Communication et organisation [En ligne], 39 | 2011, mis en ligne le 01 juin 2014, consulté le 19 avril 2019. URL : http:// journals.openedition.org/communicationorganisation/3081 ; DOI : 10.4000/

communicationorganisation.3081 


\title{
La sémiotique de terrain aujourd'hui, enjeux et propositions
}

\author{
Anthon Y̦ Mathé
}

Témoigner de la portée de l'héritage de Jean-Marie Floch, figure tutélaire de la sémiotique de terrain, comme de l'actualité de tout le champ sémiologique ${ }^{2}$ en information-communication, n'a de sens à nos yeux que si l'on montre comment il est possible d'avancer sur la route héritée, sans rien perdre de l'audace de nos maîtres. Face aux options de continuité dogmatique et de la table rase, une voie moyenne est en effet possible, qui consiste en un dialogue raisonné et une continuité récursive qui conduisent l'analyste à reconfigurer les conditions de sa praxis analytique en tenant compte de perspectives et d'orientations nouvelles. Puisque théorie et pratique fonctionnent de concert dans un rapport de co-construction, nous prenons ici le parti d'une approche contre-intuitive : plutôt que de segmenter les ordres de problème concernant la sémiotique de terrain, nous proposons de les affronter tous ensemble, de façon successive et pour ainsi dire, cumulative. Statut épistémologique de la sémiotique de terrain, rapport entre théorie et pratique, question des applications et de l'opérationnalité, circulations des savoirs sémiotiques en entreprise, perspective didactique sont autant de facettes d'un même questionnement sur les applications de la sémiotique à la communication des organisations.

Bien conscient que ces différentes facettes mériteraient un développement autonome et plus étoffé, nous sommes pourtant convaincu de la nécessité de les faire vivre ensemble car cette interaction constante entre des ordres de

\footnotetext{
1. Centre de Recherches Sémiotiques, Université de Limoges, anthony.julien.mathe@gmail.com. Docteur en sciences du langage et en sémiotique, diplômé en sciences de l'information et de la communication, chercheur associé au Centre de Recherches Sémiotiques et chargé de cours au Celsa, Anthony Mathé étudie la communication marchande et les pratiques médiatiques dans une perspective à la fois langagière et communicationnelle. Parallèlement à son activité d'enseignement et de recherche, il dirige la société Sémiolab, structure coopérative à la croisée du conseil et de la recherche universitaire.

2. Par " champ sémiologique », nous désignons non seulement les travaux issus de la sémiotique de l'École de Paris (Greimas, Fontanille), mais encore les travaux de l'École de Tartu (I. Lotman) comme les travaux en filiation avec Barthes (C. Metz, M. Joly, A. -M. Houdebine) qui ont tous en commun d'être issus du projet formulé par Ferdinand de Saussure.
} 
problèmes distincts est définitoire de la pratique de terrain. Après une brève ouverture sur la sémiotique de terrain héritée de Floch pour montrer comment avancer à partir de Floch sans reconduire ses modèles, nous proposerons une réflexion épistémologique sur le double statut de la sémiotique de terrain, à la fois expertise et recherche. Nous exposerons alors une analyse des circulations sémiotiques en entreprise à partir de témoignages recueillis sur notre propre terrain (industrie cosmétique et agences). Pour finir, dans une perspective didactique, nous effectuerons des propositions méthodologiques qui tiennent compte de l'impact de la pratique en entreprise sur la théorie de l'information-communication.

\section{La sémiotique de terrain, un héritage à rejouer}

La sémiotique de l'École de Paris a trouvé en Jean-Marie Floch un chercheur audacieux qui s'est aventuré en entreprise pour pousser plus loin l'investigation sur « la vie des signes au sein de la vie sociale ». Dix ans après sa disparition, Floch reste une référence majeure pour tout sémioticien et pour tous les acteurs du champ sémiologique tant son héritage théorique et ses apports pratiques sont une ressource vivante pour tous ceux qui s'intéressent aux phénomènes du sens et à l'analyse des pratiques communicationnelles. Reste à comprendre comment faire vivre cet héritage.

\section{Sur les pas de Jean-Marie Floch}

La manière dont on arrive en sémiotique est un moment fondateur dont la portée est considérable. Agissant comme un révélateur, le premier auteur lu reste un référent à partir duquel (ou contre lequel) le sémioticien en devenir se construit, réagit, compare, bifurque et avance. Notre intérêt pour la sémiotique - et tout particulièrement pour la sémiotique post-greimassienne a une source bien identifiée, une date précise : la lecture de Sémiotique, marketing et communication ${ }^{3}$ et d'Identités visuelles ${ }^{4}$.

Si des auteurs comme Roland Barthes, Georges Péninou ou Martine Joly constituent une mise en bouche savoureuse pour passer au plan de l'analyse, Floch va plus loin dans l'application : (1) en se donnant un terrain circonscrit et (2) en affrontant les problèmes « concrets » de ses interlocuteurs.

C'est ainsi qu'il assure l'applicabilité et l'opérationnalité des recherches sémiotiques en allant là où le porte la demande sociale. Dès lors, il illustre pleinement l'exigence hjelmslévienne d'adéquation de la théorie sémiotique avec les données de l'expérience ${ }^{5}$.

Chaque essai montre concrètement comment une théorie immanente-mais non immanentiste - telle que la sémiotique héritée de Greimas peut défendre

3. Jean-Marie Floch, Sémiotique, marketing et communication. Sous les signes, les stratégies, Paris, PUF, coll. « Formes Sémiotiques», 1990.

4. Jean-Marie Floch, Identités visuelles, Paris, PUF, coll. « Formes sémiotiques», 1995.

5. Louis Hijelmslev, Prolégomènes à une théorie du langage, Paris, Minuit, 1966. 
un empirisme méthodologique nécessaire et utile à l'étude des objets communicationnels et des processus signifiants liés aux organisations.

La filiation avec cet auteur situe de fait notre pratique sémiotique et communicationnelle dans la perspective d'une " anthropologie de nos modes d'expériences du signe et du sens ${ }^{6}$ " dont l'originalité est de considérer l'entreprise à la fois comme un terrain anthropologique et comme un objet d'étude : « [le monde de l'entreprise], en tant qu'objet d'analyse, peut représenter un lieu de mise à l'épreuve sévère et salutaire de nos hypothèses, de nos modèles et de nos procédures ${ }^{7}$ », comme l'explique Floch.

Ce souci porté aux entreprises, aux médias et aux organisations non-commerciales, a motivé très tôt notre intérêt pour les approches empiriques «immergées" de la vie des signes au sein de l'entreprise ${ }^{8}$.

Toutefois, la sémiotique de terrain qui constitue aujourd'hui notre expérience personnelle est différente. Qu'il y ait filiation ne signifie pas qu'il y ait obédience : le propos de Floch est daté et situé et, en tant que tel, il est pris dans des cadres de préoccupations professionnelles et académiques qui ne sont pas les nôtres. De la sorte, la pensée de Floch ne nous semble pas imposer un héritage à reproduire tel quel mais plutôt constituer un appel à la curiosité et à la liberté d'expérimentation, à un approfondissement systématique des découvertes par un jeu d'aller-retour entre théorie et pratique. Là est l'héritage. Nul diktat en terre sémiotique.

\section{Jean-Marie Floch entre les lignes}

Avancer sur les pas de Jean-Marie Floch signifie également le lire entre les lignes. C'est en effet entre les lignes, c'est-à-dire dans le jeu des références et à partir des implicites théoriques que le renouveau peut avoir lieu et qu'une continuité récursive, sans risque de figement ou de contre-sens, peut être assumée.

Tout d'abord, lire Floch entre les lignes, c'est relire Greimas, Lévi-Strauss, Ricœur et Wölfflin. Évidence qui n’en mérite pas moins d'être énoncée. Son usage de la notion de " discours », problématique, est en effet hérité de Greimas comme nombre de modèles ; le bricolage comme praxis est emprunté à Lévi-Strauss ; son approche de l'identité doit beaucoup à l'identité narrative de Ricœur et la distinction classique / baroque empruntée à Wölfflin s'est

\footnotetext{
6. Jean-Jacques Boutaud et Eliséo Véron, Sémiotique ouverte. Itinéraires sémiotiques en communication, Paris, Hermes / Lavoisier, coll. « Forme et sens », 2007, p. 10.

7. Jean-Marie Floch, "Concevoir et manager l'espace de travail, l'apport de la sémiotique ", in Béatrice Frankel et Christiane Legris-Desportes, Entreprise et sémiologie. Analyse le sens pour maîtriser l'action, Paris, Dunod, 1999, p. 182.

8. Bien que ne l'ayant pas côtoyé contrairement à Andrea Semprini, à Erik Bertin ou à Gérald Mazzalovo (Gérald Mazzalovo, "Exemples d'applications de la sémiotique de Jean-Marie Floch à la gestion des marques ", E/C, 2007. Consulté en ligne), nous nous présentons comme sémioticien de terrain à la suite de Floch, tant sa manière d'articuler théorie et empirie est en résonnance étroite avec le projet de Saussure comme avec celui de Hielmslev.
} 
avérée très féconde, etc. Ainsi s'avère-t-il judicieux de reporter la réflexion sur les maitres pour saisir l'apport irréductible comme les limites de chaque emprunt.

Lire Floch entre les lignes à partir d'un jeu intertextuel complexe, c'est alors comprendre les ouvertures et les restrictions qui découlent de ses efforts de dialogue tantôt avec les sciences de gestion, tantôt avec l'esthétique. C'est là que l'héritage peut être remis en jeu.

Dès lors que le dialogue s'instaure avec les sciences de la communication qui s'intéressent aux processus communicationnels et aux circulations socio-médiatiques, avec les sciences du langage, tout particulièrement la sémantique interprétative ${ }^{9}$, le jeu des ouvertures et des restrictions qui sous-tend toute problématisation change de facto et oriente la recherche vers d'autres problèmes théoriques ${ }^{10}$.

Pour aller plus loin dans cette relecture, l'actualité de la recherche fondamentale en sémiotique est une ressource sur le plan de la pratique, à commencer par les approches tensives et les recherches les plus récentes sur les niveaux de pertinence et sur les rapports textes / médias / pratiques.

En somme, si l'on se demande ce que signifie " parler concrètement » des « choses de la vie ${ }^{11}$ » dans une perspective communicationnelle, Floch ne peut que nous donner une indication en nous orientant vers l'étude des objets communicationnels, non une réponse définitive : c'est toute la théorie sémiotique dans ses acquis comme dans son devenir qu'il convient d'interroger pour comprendre que l'apport de Floch tient à une forme d'inventivité méthodologique - proprement greimassienne à nos yeux - qui n'a de sens que située dans des cadres théoriques et pratiques préétablis qui l'ont alors motivée, cadres eux-mêmes soumis à une évolution et à d'éventuelles révolutions.

\section{La sémiotique de terrain aujourd'hui}

Pour mettre en perspective les possibilités d'évolution des pratiques sémiotiques de terrain, deux temps complémentaires qui fonctionnent en miroir nous sont nécessaires : (1) une réflexion épistémologique sur l'incidence du terrain sur la praxis analytique ; (2) une observation empirique des usages et des attentes sémiotiques en entreprise ${ }^{12}$.

\section{La sémiotique de terrain entre expertise et recherche ouverte}

Notre préoccupation pour le terrain est conditionnée d'emblée par ce qui s'apparente à une double adhésion : l'adhésion à une interrogation sur

9. François Rastier, Arts et sciences du texte, Paris, PUF, coll. «Formes Sémiotiques », 2001.

10. Rappelons ici, comme l'enseigne Jacques Fontanille, qu'il n'y a pas de problème sémiotique a priori puisque le monde humain est le monde de l'intelligibilité, comme le disait Greimas dès Sémantique structurale ; il n'y a donc que des problématisations sémiotiques élaborées pour répondre à des questions provenant d'autres champs disciplinaires.

11. Jean-Marie Floch, Sémiotique, marketing et communication, Ibid., p. 1.

12. Nous prenons part au séminaire « Circulations sémiotiques » organisé par le GRIPIC. 
le langage des organisations et l'adhésion à une pratique professionnelle d'études et de conseil que Floch n'est pas le seul à incarner ; Pascal Beucler ${ }^{13}$, Andrea Semprini ${ }^{14}$, Erik Bertin ${ }^{15}$, Nicolas Couégnas ${ }^{16}$, Didier Tsala Effa et Jean-Maxence Granier qui nous ont formé pour le terrain ou sur le terrain, méritent d'être cités, sans oublier Martine Versel et Jacques Fontanille dont nous sommes l'élève.

Si en dix ans de pratique nous avons pu rencontrer plusieurs témoins de la pratique professionnelle de Floch (commanditaires, collaborateurs et élèves), nous ne sommes pas en mesure de reconstruire son contexte de travail et d'expliciter l'habitus professionnel auquel il prenait part et qui expliquerait dans quelle mesure son terrain conditionnait sa pratique. C'est d'ailleurs la raison pour laquelle nous avons décidé d'interroger notre propre terrain de façon systématique, en nous méfiant des « évidences » et du « bon sens » qui sont toujours des constructions idéologiques temporaires.

Concrètement, la sémiotique de terrain renvoie à des pratiques professionnelles d'étude et de conseil bien identifiées, que l'on appelle les "études sémio ${ }^{17}$ » dans le jargon professionnel et qui ont fait l'objet d'analyses essentielles, comme par exemple l'étude récente de Gilles Lugrin ${ }^{18}$.

De plus, le bilan dressé il y a douze ans par Eric Fouquier ${ }^{19}$ a de quoi attirer l'attention. 91 personnes pratiquaient la sémiotique alors en entreprise à Paris, soit : 24 autodidactes salariés, 27 professionnels salariés ayant reçu une formation et 37 free lances parmi lesquels des universitaires. La pratique professionnelle se répartissait alors entre des sociétés d'études spécialisées qui proposaient des études sémio à part entière et des sociétés non spécialisées qui couplaient étude sémio et approche qualitative dans une même démarche.

Le distinguo proposé par Fouquier entre «société spécialisée » et «société non-spécialisée " est redoublé par deux rapports distincts à la sémiotique : dans le premier cas, la sémiotique consiste en « une manière singulière de voir et de comprendre la communication, qui conduit à observer des phénomènes distincts du sens commun " alors que dans le second, la sémiotique est « un simple outil au service du sens commun ».

Pour ce qui est de la période actuelle, plusieurs problèmes apparaissent pour qui veut analyser les pratiques : la confidentialité, la multiplicité des acteurs, le flou entourant le terme "sémio ». Face à ces difficultés, nous proposons

13. Pascal Beucler, "Le vertige de Narcisse", Médias Pouvoirs, n42, 2ème trimestre 1996.

14. Andrea Semprini, La marque, Paris, PUF, coll. «Que sais-je?», 1997.

15. Érik Bertin, "Penser la stratégie dans le champ de la communication", Limoges, Pulim, Nouveaux Actes Sémiotiques, $n^{\circ} 110,2003$.

16. Nicolas Couégnas et Erik Bertin (sous la dir.), Solutions sémiotiques, Limoges, Lambert-Lucas, 2005.

17. Christine Legris-Desportes, Pascale Capron, Patrick Couton-Wyporek et Didier Tsala Effa, Études "sémios " et enquêtes en entreprise, Paris, Les 2 Encres, 2008.

18. Gille Lugrin, "Instrumentalisation de la sémiotique au service de la publicité et du marketing : état des lieux », in Market Mangament, 2006/4, vol. 6, pp. 5-35.

19. Éric Fouquier, "Petite histoire de la sémiotique commerciale en France », in Béatrice Frankel et Christiane Legris-Desportes, Entreprise et sémiologie, Paris, Dunod, 1999, p. 7. 
de retourner le problème en adoptant une perspective épistémologique. Il s'avère en effet pertinent d'établir une nuance entre sémiotique commerciale et sémiotique de terrain et de dresser sur cette base une typologie des intervenants.

Dès lors que l'on observe les applications sémiotiques professionnelles, il est essentiel de distinguer (1) les «études sémio » réalisées par des non-spécialistes (en l'occurrence, des qualitativistes, des communicants ou des consultants) des (2) études réalisées par des sémiologues / sémioticiens à proprement parler.

Le non-spécialiste réalise une étude sémio en appliquant un outil standard et pour ainsi dire «figé » par l'habitus professionnel : son étude sémio est ainsi un « contrat de lecture » (outil développé par Fouquier et Veron), un « fond de marque » (outil développé par la société Sorgem), une étude des « invariants » ou encore un carré sémiotique. L'outil fait l'étude.

Autrement dit, il suffit de connaître l'outil pour prétendre réaliser une étude sémio. C'est le cas, nous semble-t-il, de la majorité des études sémio réalisées en entreprise et en information-communication. Contrairement à ce que l'on pourrait penser, il n'y a pas concurrence entre cette pratique standardisée et les études sémio réalisées par un sémiologue car les deux pratiques vivent en parallèle et répondent à des besoins de compréhension distincts.

Le non-spécialiste ne participe pas à la sémiotique de terrain en tant que recherche appliquée, même si sa pratique, qui ne mérite ni critique ni rejet, s'inscrit dans le champ de la sémiotique commerciale et répond à des attentes récurrentes des organisations.

Pour le sémioticien, l'outil n'est évidemment qu'un moyen possible car l'étude sémio est un parcours balisé par des étapes et qui articule différents niveaux de questionnement concernant tantôt les conditions de production du sens, tantôt les conditions d'interprétation de la signification.

Le sémioticien de terrain est ainsi un technicien de l'analyse qui doit savoir adapter les modèles théoriques, les opérationnaliser en faisant preuve d'inventivité. Son intervention experte est motivée par la complexité des problèmes de sens qu'aucune grille d'analyse ne saurait résoudre. C'est le parcours réalisé qui fera sens ainsi : bien que circonscrite par une problématique et une commande, sa démarche exploratoire reste résolument ouverte.

Allons plus loin encore dans la typologie.

Parmi les sémioticiens de terrain, il s'avère opportun de distinguer les sémioticiens professionnels des sémioticiens universitaires : les uns sont des experts professionnels qui ont une formation complète et qui « [savent] ne pas couper leur pratique d'une réflexion théorique ${ }^{20}$ » faisant l'objet de conférences et de publications ; les autres sont des chercheurs et des spécialistes universitaires en sémiotique et en communication qui réalisent des études pour le compte d'entreprises. 
D'un côté, des experts ; de l'autre, des spécialistes. Comprenons que la tension se joue moins dans le rapport au savoir et à la théorie que dans le rapport à l'entreprise.

Le sémioticien professionnel, l'expert, évolue sur le terrain de l'entreprise et doit intégrer les contraintes internes de son commanditaire pour assurer l'efficace de son analyse. Il est ce que Karine Berthelot-Guiet appelle « une cheville ouvrière " ou encore " un passeur ", ce que nous faisons le choix d'appeler un expert. Il ne peut assurer la translation et l'opérationnalisation de modèles théoriques que dans la mesure où sa culture du domaine d'application et des objets étudiés intègre les conditions effectives de la production des textes, des discours, des produits et de tous les objets signifiants qui circulent.

La sémiotique de terrain est ainsi une sémiotique appliquée qui participe d'une sémiotique particulière définie, pour parler avec Jean-Marie Klinkenberg ${ }^{21}$, c'est-à-dire qu'elle s'intéresse à des objets précis et d'une façon qui ne préoccupe pas la sémiotique générale.

Expertise est certes un mot problématique qui fait l'objet d'abus notables dans le champ de l'entreprise et de la vie médiatique, mais qui a la commodité d'expliciter notre argument ${ }^{22}$.

Dans notre cas, l'expertise de terrain est de plusieurs ordres:(1) elle est liée à la connaissance scientifique d'un domaine de la vie sociale clairement circonscrit qui touche à la mode, à la beauté, au corps et au paraître et qui nous conduit à travailler très en amont sur des projets de parfum, de crème anti-âge ou de collection vestimentaire ; (2) elle est liée à la connaissance socio-sémiotique d'objets culturels spécifiques qui sont les supports de communication (identité visuelle, publicité, catalogue, site web, objets, espaces) et qui nous permet de collaborer avec des marques, des agences de communication ou des agences de design en amont ou en aval ; (3) elle est liée à la connaissance du métier de directeur d'études et de consultant qui a ses propres exigences et des contraintes déterminantes sur la forme donnée à l'analyse.

Au final, trois figures se dégagent : le non-spécialiste, l'expert, le spécialiste.

Nous pouvons ainsi distinguer la sémiotique proprement marchande de la sémiotique de terrain sur des critères touchant à l'épistémè, non au caractère commandité du problème de sens investi.

De plus, si nous souhaitions être polémique, il conviendrait d'ajouter aussi la figure de l'escroc ou celle du gourou, puisque certains se croient autorisés en entreprise comme en information-communication à se présenter comme sémiologues dès lors qu'ils emploient les termes signes, codes, discours et connotations.

En définitive, nous ne pouvons nier que la sémiotique appliquée à la communication s'apparente plus souvent à une instrumentalisation sémiotique

21. Jean-Marie Klinkenberg, Précis de sémiotique générale, Paris, Seuil, coll. "Points Essais ", 1996, pp. 29-33.

22. Sémir Badir, « Note sur l'expertise en sémiotique », Congrès de l'Association Française de Sémiotique, 2008. 
standardisée qu'à une sémiotique de terrain ouverte, au sens flochien d'une recherche immergée et confrontée à la vie des organisations. Le constat peut passer pour déceptif, mais la lucidité ne doit pas masquer une réalité favorable : face à la mode actuelle du « décryptage », du « décodage » et autre « révélation » farfelue, une attente d'intelligence, de méthode et de sérieux offre à la sémiotique post-greimassienne et, au-delà, à tout le champ sémiologique, la promesse de nouvelles rencontres et de nouvelles expérimentations, pour peu que l'on sache être au rendez-vous.

\section{Les circulations sémiotiques en entreprise}

Incluant l'industrie cosmétique, les instituts d'études et les agences de communication, le terrain sur lequel nous évoluons depuis près de dix ans est un contexte spécifique de productions d'objets, de discours et de valeurs. Appréhendé du point de vue de la production, c'est un tissu d'habitus professionnels, d'attentes pragmatiques ou symboliques, de croyances et de contraintes socio-économiques.

$\mathrm{Au}$ printemps 2010, nous avons réalisé une enquête prospective en interrogeant nos interlocuteurs privilégiés, soit une cinquantaine de professionnels avec qui nous collaborons de façon récurrente. Une dizaine nous ont répondu en face-à-face ou par téléphone pour des entretiens semi-directifs et nous avons envoyé un questionnaire aux autres pour savoir comment ils avaient connu la sémiotique, comment ils la définissaient, comment ils l'utilisaient et ce qu'ils en attendaient à l'avenir.

Nous envisageons aujourd'hui de compléter l'enquête en ouvrant aux sémiologues de terrain et en élargissant le champ des commanditaires.

Pour l'heure, nous présentons ici les principales conclusions de cette enquête en mettant l'accent surtout sur les perspectives et renvoyons à plus tard pour une analyse approfondie de ce matériau.

D'emblée, les agences et les instituts semblent plus à même de définir précisément ce qu'est la sémiotique et quel en est l'intérêt :

- «La sémiologie est une bien belle et utile étude des signes avec une approche méthodique et structurée qui autorise une certaine objectivitéd'analyse (comparaison, évolution, dynamisme...)»(C., directeur de création, agence).

- "La sémiologie est une méthodologie d'analyse objective pour comprendre le message transmis par un ensemble de signes (dans leur ensemble)»(K., consultante).

- "J'utilise la sémio pour une étude exploratoire, une étude de concurrence. J'attends que le sémiologue retranscrive les discours et les valeurs qui organisent et sous-tendent un marché, une catégorie de produits. » (G., directeur planning stratégique, agence).

Nulle surprise de voir les termes " signes ", " messages ", " méthode ", « objectivité » et « valeur » apparaître dans leurs propos. Agences et instituts recourent régulièrement à la sémio et doivent en justifier l'utilité s'ils veulent 
la « vendre » au commanditaire. Dans ce cas de figure, l'agence et l'institut sont à la fois commanditaire et intermédiaire.

La compréhension des tenants et des aboutissants semble claire :

- "L'utilité de la sémio, c'est essentiellement: 1 - Maîtrise de tous les éléments d'un discours (cohérence des contenus). 2 - Aide à la conception de territoires spécifiques de création. »(C., directeur de création, agence).

- "La sémiologie permet de voir l'existant et donc d'imaginer le futur. »(G., directeur planning stratégique, agence).

Si l'idée de maîtrise est une attente qui relève du fantasme, il n'en reste pas moins que ces deux citations sont en résonance avec la théorie. Par exemple, la « cohérence » si recherchée sur le terrain découle de l'aptitude sémiotique à distinguer la cohésion structurelle, la cohérence sémantique et narrative et la congruence énonciative, trois paliers d'analyse essentiels ${ }^{23}$.

Pour certains professionnels de l'industrie cosmétique, la connaissance du paradigme théorique semble hésitante mais il est intéressant de préciser que nos interlocuteurs sont en attente d'explications théoriques et conceptuelles, voire de formations :

- "La sémiologie, c'est l'art ou la technique de rendre transparent une information quion ne voit pas mais pourtant, c'est évident. » (S., directrice d'études cosmétiques)

- "J'ai découvert la sémio quand je travaillais chez Ipsos, c'était donc dans le cadre d'une application opérationnelle, avec une utilité pratique. J'ai vu la sémio non pas comme une discipline mais comme un outil de travail et j'ai envie de mieux comprendre maintenant. »(A., directrice d'études cosmétiques).

- "La question que je me pose, c'est de savoir quandje dois appeler un sémiologue? J'ai besoin de savoir comment je vais vendre ça à mes équipes, sur quels types de problématiques. » (E., directrice d'études cosmétiques).

Les usages de la sémiotique, en agence comme en entreprise, sont doubles et peuvent se résumer en deux mots clefs : (1) la cohérence et (2) la création.

Commençons par la cohérence qui semble le souci des agences comme des industriels tant la peur de la contradiction ou de l'insignifiance est forte :

- "Avec un groupe quali, les gens que tu interviewes sont difficiles à canaliser, c'est plus compliqué de les faire réagir à la cohérence du mix marketing. Alors que le sémiologue joue vraiment ce rôle de dire que tel flacon joue l'association de tel univers avec telles valeurs et que le visuel est plus "fatal " ou "provoc » et raconte telle histoire. Le rôle du sémiologue est là, dans la cohérence et dans l'articulation entre tous ces éléments. » (M. vice-président marketing).

- "La sémio permet notamment d'éviter de juger les pubs par leur esthétique seule, et de vérifier le sens des images et des mots en cohérence au brief, à l'objectif. " (D., directrice innovation cosmétique). 
La difficulté toucherait à la capacité d'évaluer la cohérence d'un projet, d'une part, au regard de la multiplicité des systèmes langagiers utilisés et, d'autre part, au regard des intentions, mais encore au regard des effets de sens possibles en contexte. Attente organisationnelle qui touche au réalisme et qui révèle le rôle médiateur de la sémiotique face aux tensions qui animent les pratiques au sein des entreprises.

Si de nombreux professionnels rappellent que ces usages « en aval » sont limités et standardisés ("On l'utilise hélas comme outil de post-rationalisation »), la question du diagnostic en entreprise demeure fondamentale et répond à des besoins organisationnels importants :

- "En entreprise, c'est un outil pour analyser précisément les signes et le sens, outil qui répond à des questions précises de la part des commanditaires. C'est un outil de diagnostic, un outil qui permet de formuler des propositions et des pistes d'amélioration...» (J., consultant).

- "L'apport de la sémio, c'est une prise de recul. C'est un point de vue extérieur et plus neutre qui nous permet d'évaluer à toutes les étapes de production nos projets et nos idées. » (E., directeur d'étude, groupe cosmétique).

Être du côté de la production, c'est être immergé dans un système qui peut faire perdre distance et perspicacité. La position « neutre » du sémiologue, construite par la méthode, est alors un atout pour évaluer la conception ou l'avancée d'un projet ; c'est là également une perspective à creuser.

Venons-en à présent à la création dont Péninou parlait déjà aux premières heures de la sémiotique de terrain ${ }^{24}$, mais qui semble faire l'objet d'attentes :

- "Ce qui m'intéresse avec la sémio, c'est de redonner du sens au signe. Il faut réintroduire une dose de créativité au milieu des schémas stéréotypés. " (JC, directeur d'agence).

- "C'est a priori une analyse et pas une source de créativité. Je ne penserais pas à utiliser un sémio en créa, et pourtant, je veux le faire... » (D., directrice innovation parfum).

- "Il y a une dimension où elle peut être utilisée, c'est comme source d'inspiration, comme point de départ, pour arriver à la genèse des idées. C'est là qu'elle devient le plus intéressant parce qu'elle est là pour mettre en évidence les rapports de force sous-jacents entre des notions, entre des concepts. Ca apporte de la vie d'un point de vue professionnel parce que nous, les pros de la communication, on travaille sur des systèmes morts. » (D, directrice planning stratégique, agence).

Passer de l'analyse à la création consiste en une passation renouvelée des résultats, en une production à part entière comme par exemple avec l'illustration des conclusions de l'analyse par l'exploration de territoires sémantiques et des recherches iconographiques, ce que les sémiologues en entreprise ont toujours

24. Georges Péninou, Intelligences de la publicité, Paris, Laffont, 1972, p. 22 : " [La sémiotique] ne dicte pas de règles de création. Simplement, plus le degré de conscience d'un créateur à l'égard de ce qu'il fait grandit, plus sa lucidité s'accroît, plus sa maîtrise du signe publicitaire peut s'affirmer, plus sa conscience de la responsabilité des signes peut se développer. » Cité par Gilles Lugrin, p. 10. 
fait à la suite de Péninou. C'est donc moins la créativité au sens fort qui compte que l'opérationnalité du résultat d'analyse, c'est-à-dire son caractère tangible.

D'ailleurs, les reproches concernent l'accessibilité des résultats, le manque de concret :

- "Idéalement,j'attends que l'étude sémio ne soit pas du "jus de cerveau". Qu'elle soit accessible. Les résultats sont parfois difficiles à s'approprier. L'opérationnalité des résultats n'est parfois pas immédiate et il y a un retravail nécessaire à faire. " (E., directeur d'études cosmétiques).

- "Le risque, c'est parfois d'être un peu trop élevé, un peu trop intello... Être plus simple, c'est bien aussi? " (M., vice-président marketing).

Une sémioticienne en entreprise analyse ainsi le dilemme que rencontre tout sémioticien de terrain : «le souci à mon sens c'est la vulgarisation des résultats pour rendre l'étude comprébensible par tous. Plus on enlève du spécifique pour rendre l'étude abordable, plus on retire en fait le fruit, les pépites qu'il faut retenir et qui se jouent dans les détails, dans les nuances de sens. " Il serait bon de rappeler que la valeur de l'analyse sémiotique touche à sa technicité conceptuelle et méthodologique et que le talent du sémiologue est d'arriver à concilier cette technicité avec l'exigence de simplicité qui, rappelons-le, constitue un principe sémiotique fondamental selon Hjelmslev ${ }^{25}$.

Retenons de ces verbatims d'entretiens deux couples de notions : (1) l'objectivité et la neutralité liées à la quête de cohérence ; (2) la co-construction et l'accompagnement qui précisent les attentes de créativité :

- "C'est sa capacité de neutralité qui importe." (A., directrice études cosmétiques)

- "La sémiologie permet de mettre de l'ordre dans le foisonnement de réactions que tout en chacun peut avoir vis-à-vis d'un objet : flacon, communication, nom, odeur, dans mon cas. Ga priorise, ça met en avant les logiques profondes sous-jacentes très objectives et donc ça met tout le monde d'accord puisque c'est objectif. En plus, ça permet de mieux briefer et comprendre les études qualitatives sur les mêmes stimuli. Pour moi c'est une science très fiable et utile, et aussi un outil diplomatique très précieux.» (C., directrice études cosmétiques).

- "J'attends du sémiologue un rôle important d'accompagnement, avec cette démarche critique par rapport au marketing. Le rôle du sémio est d'être le plus objectif possible, justement parce que dans l'équipe marketing, on est le moins objectif, on est instinctif. Le sémiologue peut alors t'accompagner dans un rôle de coordination et d'articulation. Ce qui est fondamental dans cette perspective, c'est de comprendre les objectifs et tout doit partir de ça. » (M., vice-président marketing, groupe cosmétique).

En résumé, distance, neutralité, objectivité, accompagnement, co-construction et créativité sont les mots clefs pour comprendre les attentes de nos interlocuteurs quant au diagnostic sémiotique. Arrêtons-nous ici

25. Louis Hielmslev, "Entretien sur la théorie du langage », in Nouveaux Essais, Paris, PUF, coll. " Formes sémiotiques », 1985, p. 73. 
sur son rôle « diplomatique » qui constitue toute sa pertinence comme la clef de son devenir à nos yeux. Cette vision de la sémiotique comme « outil diplomatique " révèle quelque chose d'essentiel : dans des organisations soumises à des tensions internes très variées, parfois contradictoires et polémiques, et à des pressions concurrentielles, la sémiotique semble à même fournir une médiation théorique et méthodologique sous la forme d'un discours critique neutre et intermédiaire qui ne donne pas seulement du recul, mais surtout des moyens de traduire le point de vue de chacun dans une vision d'ensemble partageable. D'où la possibilité d'un dialogue constructif à partir d'une neutralisation des tensions. Ce rôle de médiation par la sémiotique existe également dans les relations entre disciplines des $\mathrm{SHS}^{26}$, mais quand il s'agit de l'entreprise et des pratiques organisationnelles, nous constatons combien ce dialogue entre positions et points de vus concurrents ou contradictoires est une force irréductible de la sémiotique.

Au regard de la théorie, il nous semble que la neutralité (la posture construite), la technicité (le savoir-faire opératif) et l'accompagnement (le souci d'adéquation et l'écoute) constituent les trois pôles de l'expertise sémiotique pour parvenir à faciliter le dialogue et à traduire les points de vue de tous en un langage commun. La triangulation entre ces trois facettes permet d'identifier des perspectives de réflexion quant aux conditions d'une sémiotique de terrain en communication.

Soit le schéma suivant :

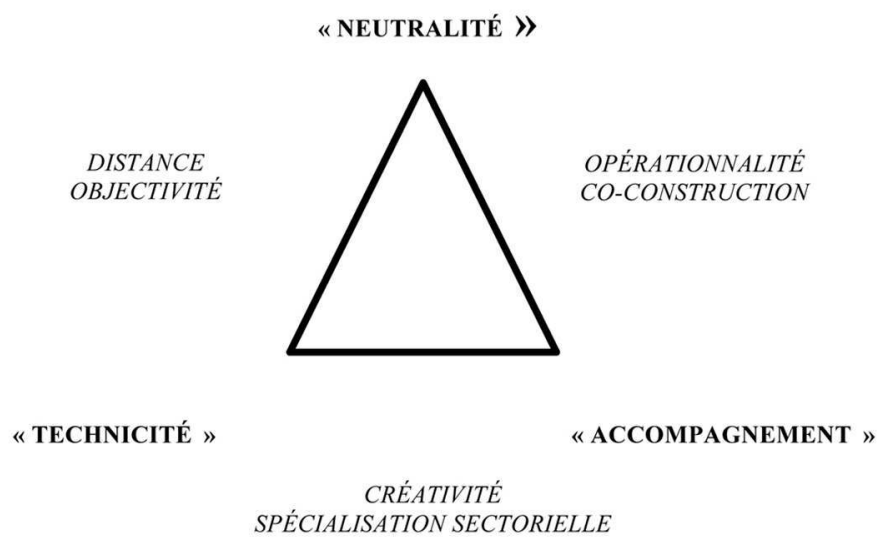

Les trois piliers de l'expertise sémiotique de terrain

À défaut de pouvoir aborder en détail ici tous les points mentionnés et qui sont pourtant essentiels, nous proposons de nous focaliser pour l'heure sur les rapports entre neutralité et technicité et de partager des propositions

26. Sémir Badir, "Pour une sémiotique indisciplinée », Les signes du monde. Interculturalité et globalisation, Actes du Congrès de l'Association internationale de sémiotique, Lyon, 2004. 
méthodologiques à visée didactique élaborées à partir du terrain qui permettent de concrétiser ce rôle de médiation joué par la sémiotique au sein des organisations.

\section{Le renouveau sémiotique, propositions méthodologiques}

Pour faire retour de la pratique à la théorie, nous proposons de partager deux protocoles d'analyse adaptés aux problématiques communicationnelles et qui constituent le cœur de nos enseignements à l'université.

Sans hésiter, nous prenons le parti d'une approche pragmatique réfléchie qui privilégie les modes de questionnement aux outils : ce sont les interrogations langagières et les problématisations communicationnelles qui font sens à nos yeux ; qu'ils soient des productions théoriques nécessaires ou des constructions empiriques utiles, les outils nous inspirent une certaine méfiance car l'instrumentalisation est un risque qu'il importe de déjouer en amont.

Comme nous le savons, la théorie sémiotique vise à rendre compte des conditions de production et d'interprétation de la signification. À partir de ce double enjeu, la dissociation temporaire des perspectives d'analyse permet d'articuler deux protocoles d'analyse, l'un concernant les conditions de production de la signification, l'autre, les conditions de son interprétation.

\section{Les quatre étapes clefs de l'analyse des objets communicationnels}

Pour ce qui est de l'analyse des conditions de production du sens des objets communicationnels, notre exigence première est de concevoir un protocole d'analyse à la fois suffisamment général pour objectiver les pratiques organisationnelles et suffisamment spécifique et technique pour s'appliquer aux supports communicationnels. Telle une trame minimale, ce protocole est un questionnement ouvert sur les formes textuelles et les formes de vie qui circulent au sein des pratiques communicationnelles. Nous l'avons ainsi développé à partir du terrain et des questions récurrentes qui nous sont posées.

Pour répondre ainsi aux exigences de technicité, de simplicité et de neutralité, notre protocole d'analyse permet de décrire, d'examiner et d'évaluer progressivement toutes données et de montrer comment les récits se construisent et se modifient :

\section{Expression:}

Une analyse en monadique des documents procède par étapes pour rendre compte de ses différentes composantes en soi selon l'ordre suivant : structure, plastique, thématique, sémantique et sémio-narrativité avant de voir leur rôle " en contexte », une fois rapporté au support utilisé.

\section{Comparaison:}

Puisque le sens naît de la différence, la prise en compte de données équivalentes (concurrence, benchmark, comparaison hors secteur) permet 
d'établir une paradigmatisation du domaine et de confronter concrètement les documents à d'autres formes et d'autres pratiques de production.

\section{3. Énonciations:}

L'analyse du projet rapportée à la marque procède en plusieurs temps qui consistent à moduler le point de vue en s'intéressant successivement (1) à l'organisation ${ }^{27}$ et à son image ${ }^{28}$ (son histoire, son patrimoine, son cœur de métier, sa légitimité, sa culture d'entreprise), (2) à la forme-marque en tant qu'instance de discours (son monde possible, son dispositif communicationnel) et (3) à l'être culturel ${ }^{29}$ (ses circulations socio-médiatiques, ses appropriations multiples par les cibles et sa co-construction avec les publics).

\section{Cultures:}

L'analyse rapportée aux pratiques sociales afférentes et au contexte culturel correspond à un questionnement sur le sens commun et sur la valeur sociale des données ; c'est le moment de se poser la question du pourquoi au-delà du comment et de confronter les documents aux pratiques sociales; c'est le moment aussi où il convient d'interroger les évocations susceptibles d'être associées et les connotations socioculturelles à l'aide de dictionnaires, de recherches documentaires et en faisant appel à des spécialistes de chaque culture, selon les besoins.

À défaut d'illustrer l'application de ce protocole, nous soulignerons que sa pertinence tient au parti méthodologique de segmentation et d'articulation des ordres de problèmes, ce qui répond tant aux demandes sociales qu'aux exigences épistémologiques. Notre protocole contourne le hiatus inéluctable existant entre les modèles sémiotiques et les données étudiées : chaque modèle sera ici convoqué, si besoin, à partir des objets et au sein de questionnements préétablis qui circonscrivent les objectifs d'analyse.

Précisons rapidement que ce protocole n'a pas simplement été élaboré à partir des questions rencontrées sur le terrain. Il découle aussi d'une opérationnalisation du modèle épistémique des niveaux de pertinence proposé par Jacques Fontanille qui structurent chaque parcours d'analyse en paliers successifs et permettent ainsi de se confronter à des phénomènes de sens qui sont ni textuels ni textualisables ${ }^{30}$. Pour étudier les pratiques communicationnelles, nous avons tant besoin d'étudier l'expérience des textes, des images et des objets, que des supports, des médias, des pratiques de lecture et de réécriture, et des circulations. Il est clair que l'usage du protocole s'en tient à trois paliers culturels qui nous paraissent suffisants ici : le texte et son support, l'instance de discours, la pratique culturelle associée.

27. Valérie Carayol, Communication organisationnelle : une perspective pragmatique, Paris, L'Harmattan, 2004.

28. Gilles Marion, Les images de l'entreprise, Paris, Les Editions d'Organisation, 1989.

29. Yves Jeanneret, Penser la trivialité. Volume 1. La vie triviale des êtres culturels, Paris, Hermès Lavoisier, 2008.

30. Jacques Fontanille, Pratiques sémiotiques, Paris, PUF, coll. « Formes Sémiotiques », 2008. 


\section{Les scénarios interprétatifs}

Une fois effectués les quatre temps del'analyse des objets communicationnels, la question de la synthèse et de l'interprétation se pose inéluctablement.

Pour opérationnaliser le principe d'interprétation conditionnelle, nous proposons de recourir à des scénarios interprétatifs, c'est-à-dire de formuler des hypothèses d'interprétation sur les objets communicationnels et qui concernent les paliers que sont le texte, la marque, la pratique culturelle. Deux usages des scénarios interprétatifs sont possibles.

Le premier usage suppose de reconsidérer l'analyse en monadique d'un objet communicationnel. Prenons l'exemple d'un mix marketing dans l'industrie du parfum, soit : un nom, une marque, un flacon, un packaging, un jus, une publicité. Chaque élément sera analysé séparément.

Lidée du scénario interprétatif est de voir comment les éléments se combinent et se modulent réciproquement. Pour cela, nous procédons par étapes cumulatives : nom + flacon; puis, nom + flacon + marque ; puis, nom + flacon + marque + publicité, etc. L'idée est de voir comment le sens émerge et se module en fonction des éléments en présence. L'analyse segmentée et séquencée, présentée telle quelle, répond à une attente de technicité des demandes organisationnelles facile à satisfaire; il ne s'agit que d'organisation. De plus, nous explorons tous les autres scénarios possibles : flacon + publicité ; flacon + packaging, etc. Nous comparons des scénarios proches: flacon + publicité versus flacon + publicité + marque. C'est ainsi que l'analyse du projet permet de rendre compte des niveaux expérientiels de sa signification. Chaque scénario interprétatif permet de réévaluer tous les contenus d'analyse et d'identifier éventuellement les éléments susceptibles de paraître saillants dans telle ou telle autre configuration de lecture.

Le second usage des scénarios interprétatifs concerne tous les paliers de notre protocole et repose sur un constat de terrain : les scénarios interprétatifs gagnent en pertinence dans le cadre d'une co-construction avec ses interlocuteurs, quels qu'ils soient. Comme le suggèrent nos interlocuteurs, l'intégration de variables non-sémiotiques, pratiques, contextuelles ou économiques, permet de relancer et de compléter le parcours d'analyse :

- "Pour toute étude sémio, ce serait bien que le sémiologue soit briefé de manière très précise sur ce qui marche ou pas actuellement dans le marché concerné. Et ça, c'est le next-step pour moi. C'est l'avenir de la sémio. Parfois, il y a une analyse très positive d'une communication qui ne marche pas. Peut-être que si le sémiologue savait qu'elle ne marche pas, il se poserait d'autres questions. " (C., directrice études cosmétiques)

- "L'approche sémio gagne en pertinence s'il y a prise en compte du social, de ses questionnements, de ses usages, de ses interprétations empiriques, etc. La sémio est alors très fructueuse pour expliquer ce qui est en jeu. » (J., consultant).

La prise en compte de "l'intention ", de "la réception » et des usages sociaux aide à démultiplier les parcours interprétatifs plausibles. Les scénarios 
peuvent tout aussi bien être élaborés à partir d'informations statistiques, sociologiques ou culturelles. Pour moduler les hypothèses de lecture, on peut également jouer sur des variables triviales comme la connaissance (" si le lecteur connaît..., alors... "; " si le lecteur ne connaît pas, alors... ») ; le jugement de valeur ("s'il aime ou s'il n'aime pas..., alors...»), l'opinion ou tout ce qui sera jugé pertinent. Pour simple qu'elle soit, notre idée du scénario est de systématiser dans un second temps la réévaluation des parcours d'analyse effectués à partir d'un protocole adapté et facilement adaptable. C'est une façon de tester des pratiques de lecture possibles et plausibles sans sortir du domaine sémiotique.

\title{
Conclusion
}

En guise de conclusion, nous aimerions rappeler que l'irréductible apport de la sémiotique en information-communication, et au-delà, touche à une démarche atypique de médiation interdisciplinaire et à un mode de questionnement original sur les phénomènes de sens, non à des réponses matérialisées sous forme d'outils. La pratique - toujours circonscrite - du sémioticien de terrain n'a de sens que si elle est une reformulation spécifique des modèles généraux et consiste en " une participation personnelle de l'analyste aux interrogations d'ensemble ${ }^{31}$ ". Ainsi, le sens du sens de la démarche sémiotique tient dans la capacité à faire évoluer les modélisations en fonction des perspectives d'application dans le cadre d'un double mouvement qui conduit de la théorie à la pratique, et du terrain à la théorie.

Résumé : Adossée à des considérations épistémologiques sur le statut de la sémiotique de terrain, l'analyse des circulations sémiotiques en entreprise à partir de témoignages recueillis sur notre terrain de recherche (industrie cosmétique et agences de communication) conduit à proposer des protocoles d'analyse ouverts adéquats aux questionnements information-communication et qui permettent d'envisager la contribution de la sémiotique comme une ressource méthodologique essentielle pour l'analyse des pratiques organisationnelles.

Mots-clefs : Sémiotique de terrain, communication, épistémologie, expertise, industrie cosmétique, agences de conseil, protocoles d'analyse, information-communication.

\begin{abstract}
Rooted in the epistemological considerations coming forth from the status of terrain semiotics, the analysis of semiotic exchanges in the business world, based on the gathered testimonies of our research terrain (the cosmetics industry and communication agencies), has led us to propose adequate, open analysis protocols for information-communication questionings. These protocols equally enable us to envisage the contribution of semiotics as an essential methodological resource for the analysis of organizational practices.
\end{abstract}

Keywords : Applied semiotics, epistemology, expertise, cosmetic industry, communication agency, protocol of analysis, sciences of communication.

31. Anne Hénault, Les enjeux de la sémiotique, Paris, PUF, coll. « Formes Sémiotiques », 1979, p. 174. 